\title{
Positive Spacecraft Charging as Measured by the Shuttle Potential and Return Electron Experiment
}

\author{
M.R. Oberhardt ${ }^{1}$, D.A. Hardy \\ Phillips Laboratory, GPSP, Hanscom AFB, MA 01731 \\ D.C. Thompson, W.J. Raitt \\ Utah State University, Logan, UT 84322 \\ E. Melchioni \\ Instituto di Fisica dello Spazio Interplanetario, Frascati, Italy 00044 \\ C. Bonifazi \\ Agenzia Spaziale Italiana, Rome, Italy 00198 \\ M.P. Gough \\ Space Science Centre, School of Engineering, University of Sussex, Brighton, UK BN19QT
}

\begin{abstract}
During the deployed phase of the Tethered Satellite System 1 Mission (TSS-1), the Orbiter was observed to charge positively on a number of occasions during operation of the $100 \mathrm{~mA}, 1$ $\mathrm{keV}$ Fast Pulsed Electron Gun (FPEG) of the Shuttle Electrodynamic Tether System (SETS). The occurrence of positive charging was determined both through the measurement of the voltages in the system and through observation of the charging peaks in the electron spectra measured by the Shuttle Potential and Return Electron Experiment (SPREE). Here we present data from the two cases of highest positive charging during the deployed phase of the TSS-1 mission. These cases occurred in darkness during periods of depressed ambient plasma density. Positive Orbiter charging was observed from ten to a hundred volts. During the operation of the FPEG prior to Orbiter charging, the SPREE electrostatic analyzers measured intense fluxes of electrons at energies up to the energy of the emitted beam. During the charging periods, the SPREE electron spectra displayed a peak whose position in energy was consistent with the positive potential of the Orbiter as determined from SETS and the Italian Space Agency (ASI) Deployer and Satellite Core Equipment (DCORE and SCORE) measurements. At energies above the charging peak, the shape of the electron distribution function during charging was consistent with a simple acceleration of the pre-charging spectrum by the electric field produced by the positively charged Orbiter. At energies below the charging peak, intense, isotropic fluxes of electrons were measured either with a power law spectrum or with a spectrum peaked at 10 to $20 \mathrm{eV}$.
\end{abstract}

\section{INTRODUCTION}

The Shuttle Potential and Return Electron Experiment (SPREE) was flown in the Orbiter payload bay as an integral part of the joint NASA/Italian Space Agency (ASI) TSS-1 mission. The TSS-1 mission was conducted during the STS-46 flight of the Space Shuttle Orbiter, launched on July 31, 1992. For this mission, the Orbiter was in a $28^{\circ}$ inclination orbit. The TSS-1 deployed activities were conducted at a $295 \mathrm{~km}$ altitude. The goal of the TSS-1 mission was to investigate the mechanical and electrical dynamics of a conducting satellite deployed from the Orbiter by a conducting tether. For TSS-1, the satellite was to be deployed from the Orbiter to a maximum distance of 20 kilometers. Both the TSS-1 satellite and the Orbiter carried instruments designed to study the electrodynamic processes.

In the TSS-1 system, the satellite is deployed by the conducting tether from the Orbiter radially away from the earth. In this configuration the tether is moving relative to the earth's magnetic field. As a result, a motional electromotive force $(e m f)$ is produced that is a product of Orbiter velocity, the Earth's magnetic field, and the length of the deployed tether,

$e m f=\left(\overrightarrow{\mathrm{v}}_{\text {Orbiter }} \times \overrightarrow{\mathrm{B}}\right) \cdot \overrightarrow{\mathrm{L}}$

For the STS-46 orbit, the magnitude of the emf averaged $0.22 \mathrm{~V} / \mathrm{m}$. At full deployment to the planned distance of 20 $\mathrm{km}$, a potential drop between the satellite and the Orbiter of up to $5 \mathrm{kV}$ would have been generated. As a result of this emf, a current can be driven in the tether. The electrically conductive

\footnotetext{
${ }^{1}$ Now at Space Sciences Laboratory, University of Califomia, Berkeley, CA
} 
satellite skin conductive acts as an anode in the TSS-1 circuit, collecting electrons that could travel along the conducting tether to the Orbiter where they could either be routed to Orbiter structural ground or be emitted into the ionosphere via active electron emission.[1,2,3,4,5] The total potential drop across the tether is equal to the sum of all of the potential drops in the system,

$\left(\vec{v}_{\text {Ortiter }} \times \vec{B}\right) \cdot \vec{L}-I_{\text {temer }} R_{\text {lecher }}=\phi_{\text {Orbiter }}+\phi_{\text {satellite }}+\phi_{\text {plasma }}$

with $\mathrm{R}_{\text {tether }} \approx 2 \mathrm{k} \Omega$, and $\phi_{\text {plasma }}$ the potential drop in the ionosphere.

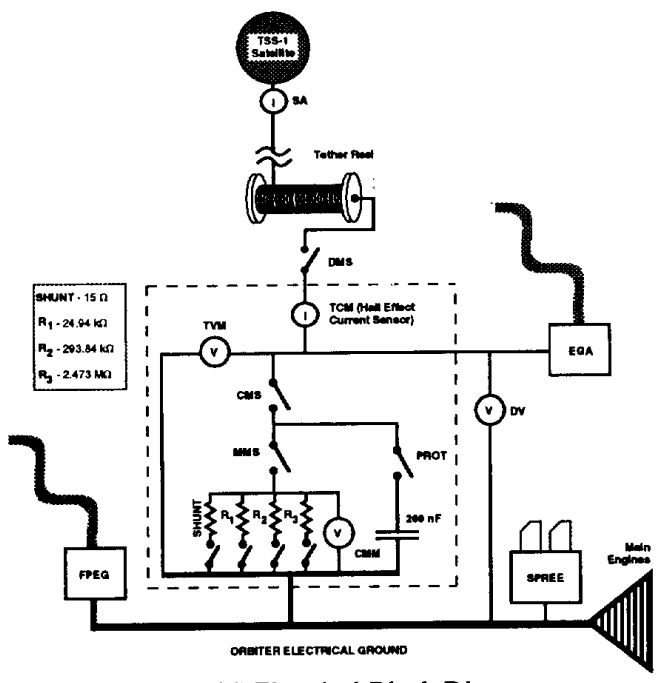

Figure 1 TSS Electrical Block Diagram

As precursors to TSS-1, numerous rocket-borne electrodynamic tether experiments have illustrated the usefulness of tethered systems in the study of space plasma phenomena. $[6,7,8,9,10,11,12]$ The TSS-1 system provided the first orbital platform of a tethered system for such studies. This system could be configured in a number of modes. In one mode, the tether current is routed to the Orbiter ground either directly (through a shunt resistor of $15 \Omega$ ) or through a known, larger resistance. (See Figure 1.) This routing is controlled in the SETS Tether Current Voltage Monitor (TCVM). In this configuration, the Orbiter and TSS satellite charge to the voltages necessary to balance the current collection at the two locations. The Orbiter would charge negatively and the satellite positively. In another mode, the tether again is connected to Orbiter ground via the TCVM, but in this case the SETS Fast Pulse Electron Gun (FPEG) is used to emit the collected electrons into the ambient plasma.[4,13,14,15] A third mode has the tether electrically connected to an electron beam system isolated from the Orbiter ground. This beam system, the ASI Deployer Core Equipment (DCORE) Electron Gun Assembly (EGA) is used to complete the TSS-1 current loop to space.[16,17] A fourth mode has the satellite electrically isolated through a large impedance from the Orbiter, with essentially no current flowing and no electron beam emission, allowing the satellite to act as a reference electrode and the scientific instruments to measure the ambient plasma.

Many theoretical studies have been conducted on current collection by a positive probe in a space plasma[18]. These works have included charging of vehicles emitting electron beams. Such charging has been shown to be controlled by either space charge effects[19] or magnetic field effects[20] that act to limit ionospheric return currents to the vehicle during electron beam emission. Comparisons of flight data to these theories are not straightforward given that the objects theoretically modelled in these works were simple spheres and not actual spacecraft shapes. Also, the electron beam can be backscattered, produce secondary emission from surfaces it strikes, and cause ionization, making modelling difficult. The results reported here offer new insight into that return current collection process while the modelling effort to fully quantify this process continues in light of these new data.

\section{INSTRUMENTATION OVERVIEW}

The key elements of the SPREE system are two multiangular electrostatic analyzer (ESA) units each mounted on a rotary table (RTMD). Each ESA measures the flux of electrons and ions in 32 logarithmically spaced energy channels covering the range from $10 \mathrm{eV}$ to $10 \mathrm{keV}$. The particles are measured in an angular fan, of $100^{\circ} \times 10^{\circ}$. Within the $100^{\circ}$ dimension of the fan particles are measured in ten, $10^{\circ}$ zones numbered 0 to 9. The center of the angular range of Zone 0 is parallel to the ESA mounting surface while that for Zone 9 is towards the zenith. The ESAs are mounted on rotary tables in order to measure over a full $2 \pi$ steradian solid angle out of the Orbiter bay at the SPREE location. Each rotary table mechanically sweeps an ESA through $180^{\circ}$ in azimuth at a rate of approximately $6^{\circ}$ per second. In normal operation, the rotary tables' motions are synchronized with the ESAs' detection fans $180^{\circ}$ apart. This permits coverage of the full $2 \pi$ steradian every 30 seconds. The ESAs have two modes of operation, returning either one complete spectrum per second (slow mode) or eight complete spectra per second (fast mode). The geometric factors for the two ESAs differ by approximately two orders of magnitude, generally allowing unsaturated measurements of the ambient particle population during both quiescent and perturbed plasma conditions, even for direct return of electron beam particles to the instrument.[21,22,23]

SPREE included a Spacecraft Particle Correlator Experiment (SPACE) to measure modulations of the electron and ion fluxes as might be produced by coherent wave-particle interactions. The heart of the SPACE electronics package is a set of microcontrollers and hardware processors, all controlled by a microprocessor. The key inputs to the SPACE are the SPREE particle detection pulses from the 40 angular zones of the detector. SPACE uses the high frequency clock in the microprocessor to time the arrival of each pulse to the accuracy 


\begin{tabular}{|ll|}
\hline & \multicolumn{1}{c|}{ Table I } \\
& \\
ASI & AgS-1 Acronyms \\
CMM & Current Mode Monitor \\
DCORE & Deployer Core Equipment \\
DEP1 & Deployment 1 Cycle \\
DMS & Deployer Master Switch \\
DV & DCORE Voltmeter \\
EGA & Electron Gun Assembly \\
ESA & Electrostatic Analyzer \\
FES & Flash Evaporator System \\
FPEG & Fast Pulsed Electron Gun \\
LF & Low Frequency \\
MMS & Mechanical Mode Switch \\
RCS & Reaction Control System \\
RETE & Research on Electrodynamic Tether \\
& Experiment \\
RTMD & Rotary Table Motor Drive \\
SA & Satellite Ammeter \\
SCORE & Satellite Core Equipment \\
SETS & Shuttle Electrodynamic Tether System \\
SPACE & Spacecraft Particle Correlator \\
SPREE & Experiment \\
TCM & Shuttle Potential and Return Electron \\
TCVM & Experiment \\
TSS-1 & Tether Current Monitor \\
TVM & Tether Current and Voltage Monitor \\
& Tethered Satellite System 1 \\
\hline
\end{tabular}

of the period of the clock. Utilizing a number of signal processing techniques, SPACE can analyze a pulse stream for either high frequency $(0-10 \mathrm{MHz})$ or low frequency $(0-10 \mathrm{kHz})$ modulations.

In this study we present only data from the SPACE Low Frequency (LF) Autocorrelator. In the LF Autocorrelator, true autocorrelations are performed in the frequency range from 0 $10 \mathrm{kHz}$ for both ions and electrons. In this technique, the particle generated pulse trains are analyzed separately in each SPREE zone and at each energy channel. In each such case, the pulses are processed for a selected time interval. The duration of the interval is set according to the frequency range to be studied. The interval is divided in 64 bins of equal width in time. The number of pulses observed in each bin is stored. At the end of the interval, this set of 64 numbers is autocorrelated to generate a 32 point autocorrelation function (ACF) that is summed at a given energy and zone over the interval. A coherent modulation of the particles will appear in the ACF as a series of periodic peaks.[22]

Data for this study were also provided by SETS, the Research on Electrodynamic Tether Experiment (RETE), and the DCORE \& SCORE. The SETS data were from the FPEGs and the TCVM. The FPEG emitted a $100 \mathrm{~mA}, 1 \mathrm{keV}$ beam in either a $\mathrm{DC}$ mode or in an $\mathrm{AC}$ mode, with a pulsing range from DC up to $1.25 \mathrm{MHz}$. Note that when an ESA's azimuthal position is $180^{\circ}$, it is viewing along the direction of FPEG emission. As previously noted, the TCVM controlled the path of the tether current at the Orbiter. In addition, the TCVM measures the tether current using a Hall effect current sensor and the tether potential when the circuit is open. The RETE consisted of a set of wave receivers and thermal plasma probes on the TSS satellite.[24] The RETE measurements used in this study include the potential of the satellite, the local plasma potential, the electron temperature, and the ion and electron densities, all obtained from the DC Boom Package (DCBP) on the satellite. Accurate tether current and voltage measurements were provided by the DCORE and SCORE.

\section{POSITIVE ORBITER CHARGING CASES}

A number of periods of positive charging of the Orbiter occurred during the deployed portion of the TSS-1 mission with the FPEG operating. These positive charging events were primarily determined from TCVM and DCORE data using one of two techniques. In the first method, the tether was electrically disconnected from the Orbiter. For this case, the satellite was isolated from the Orbiter through a large impedance and acted as a reference electrode. The TCVM and the DCORE independently measure the emf-induced potential across the tether. We know that the major contribution to this measured potential was the motional emf and the potential produced by the operation of FPEG and that other sources (e.g. potentials induced by naturally occurring ionospheric electric fields) are second order effects. Also, $\phi_{\text {plasma }}$ is assumed to be negligible in this study given the short tether length. The satellite potential will be near zero since there was no current flow in the tether, giving,

$\phi_{\text {Orbiter }}=e m f-\phi_{\text {Tether }}$

where emf is the calculated $v x B \bullet L$ voltage. The emf can be calculated from the model of the earth's magnetic field, the known orbital position and the orientation of the tether system. The difference between the calculated $v \times B \bullet L$ voltage and the measured tether potential gives the expected Orbiter ground voltage which can then be compared to the SPREE electron spectral peaks.

In the second method, the tether is connected to the Orbiter ground through one of the TCVM resistors, and the TCVM and the SCORE independently measure the tether current. When the value of the TCVM resistor is large such that the tether current is small, the potential of the satellite ground with respect to the ambient plasma is still close to zero. For this case, the sum of the voltage drop across the known tether resistance, the voltage drop across the resistor in the TCVM, and the $v x B \bullet L$ voltage must equal the voltage of the Orbiter ground (Equation 4). Since the first two are measured and the third can be calculated, the value of the Orbiter potential can be specified. Even for cases where there is a 
smaller TCVM resistor in-line, the Orbiter ground voltage can be calculated from a knowledge of the tether current and the ground potential of the satellite supplied by RETE (Equation 5). Again, the SPREE measured electron spectral peaks can be compared to this determined value of $\phi_{\text {Orbiter }}$.

$$
\begin{aligned}
& \phi_{\text {Otbiter }}=e m f-\left(\mathrm{I}_{\text {tether }} \mathrm{R}_{\text {tether }}+\mathrm{I}_{\text {tether }} \mathrm{R}_{\mathrm{TCVM}}\right) \\
& \phi_{\text {Orbiter }}=e m f-\left(\mathrm{I}_{\text {tether }} \mathrm{R}_{\text {techer }}+\mathrm{I}_{\text {tether }} \mathrm{R}_{\mathrm{TCVM}}+\phi_{\text {satellite }}\right)
\end{aligned}
$$

The charging events reported here occurred during the Deployment 1 (DEP1) operating cycle. The DEP 1 cycle consisted of four steps, each lasting roughly 100 seconds, all with the Deployer Master Switch (DMS) closed such that the tether could be electrically connected to the Orbiter through the TCVM. (See Figure 1.) The SPREE ESAs were always operating in the fast deflection sweep mode for DEP 1 cycles. SPREE data shown in this paper will be from DEP 1 Steps 1

\begin{tabular}{|c|c|c|c|c|c|}
\hline \multirow[b]{2}{*}{ Event } & \multicolumn{4}{|c|}{$\begin{array}{c}\text { Table II } \\
\text { TSS-1 Positive Orbiter Charging Cases }\end{array}$} & \multirow[b]{2}{*}{$\begin{array}{l}\text { Tether } \\
\text { Length(m) }\end{array}$} \\
\hline & GMT & $\begin{array}{l}\text { FPEG } \\
\text { Escape }\end{array}$ & $\begin{array}{l}\text { Day } \\
\text { Night }\end{array}$ & $\begin{array}{l}\text { FES/Water/Thruster } \\
\text { (F)/ (W) } /(\mathrm{T})\end{array}$ & \\
\hline 1 & $217 / 23: 32$ & No & Night & $\mathbf{T}$ & $105.3-1489$ \\
\hline 2 & $217 / 23: 46$ & No & $\begin{array}{l}\text { Night/Day } \\
\text { Terminator }\end{array}$ & $\mathrm{T}$ & 191.4 \\
\hline 3 & $217 / 23: 53$ & No & Day & $\mathbf{T}$ & 191.4 \\
\hline 4 & $217 / 23: 59$ & No & Day & $\mathbf{T}$ & 191.4 \\
\hline 5 & $21800: 33$ & Yes & $\begin{array}{l}\text { Day/Night } \\
\text { Terminato }\end{array}$ & $\mathrm{F}, \mathrm{T}$ & 185.5 \\
\hline 6 & $218 / 01: 01$ & Yes & Night & $\mathbf{F}, \mathbf{T}$ & 185.5 \\
\hline 7 & $218 / 02: 06$ & Yes & $\begin{array}{l}\text { Day/Night } \\
\text { Terminator }\end{array}$ & $\mathrm{F}, \mathrm{T}$ & 267.3 \\
\hline 8 & $218 / 02: 12$ & Yes & Night & F, T & 267.3 \\
\hline 9 & $218 / 02: 20$ & Yes & Night & F, T & 267.3 \\
\hline 10 & $218 / 02: 33$ & Yes & Night & F, T & 267.3 \\
\hline 11 & $218 / 02: 39$ & No & Night & F, T & 267.3 \\
\hline 12 & $218 / 02: 46$ & No & $\begin{array}{l}\text { Night/Day } \\
\text { Terminator }\end{array}$ & F, T & 267.3 \\
\hline 13 & $218 / 02: 53$ & No & Day & $\mathrm{F}, \mathrm{T}$ & 267.3 \\
\hline 14 & $218 / 23: 29$ & No & Night & $\mathrm{F}, \mathrm{T}$ & 23.3 \\
\hline
\end{tabular}
and 2.

DEP 1 Step 1 had the satellite electrically isolated from the Orbiter and the FPEG pulsing at various frequencies and duty cycles. Measurements of the Orbiter potential in conjunction with the FPEG emissions could then be made while the scientific instruments on the satellite could make measurements of the unperturbed plasma in its vicinity. In DEP 1 Step 2 the tether was connected to the Orbiter ground through the resistor/capacitor bank downstream of the Current Mode Switch while at the same time the FPEG fired a DC beam. The TCVM resistors were again cycled in Step 3 but with no FPEG emission. DEP 1 Step 4 was a passive one, with the satellite electrically isolated, no FPEG emission, and no cycling of the TCVM resistors.
Table II lists the times for which positive charging has been identified in the SPREE electron spectra along with relevant environmental information. Note that for all of these events, SPREE was in the fast deflection sweep mode. We will discuss in detail the two largest positive charging events, 217/23:32 and 218/01:01 (Events 1 and 6). For the two events presented here, the thruster firings are not correlated to triggering the charging process as they are in Events 2-5 and 7-14. Events 1 and 6 are correlated to passage of the TSS- 1 through regions of depressed ambient plasma density as measured by RETE.[25] The RETE measurements indicate that positive charging occurred for densities below $6 \times 10^{5}$ electrons $/ \mathrm{cm}^{3}$. To facilitate identification of data features in the figures that follow, Table III is included and lists the start and end times for the steps of the Event 1 and Event 6 DEP 1 cycles.

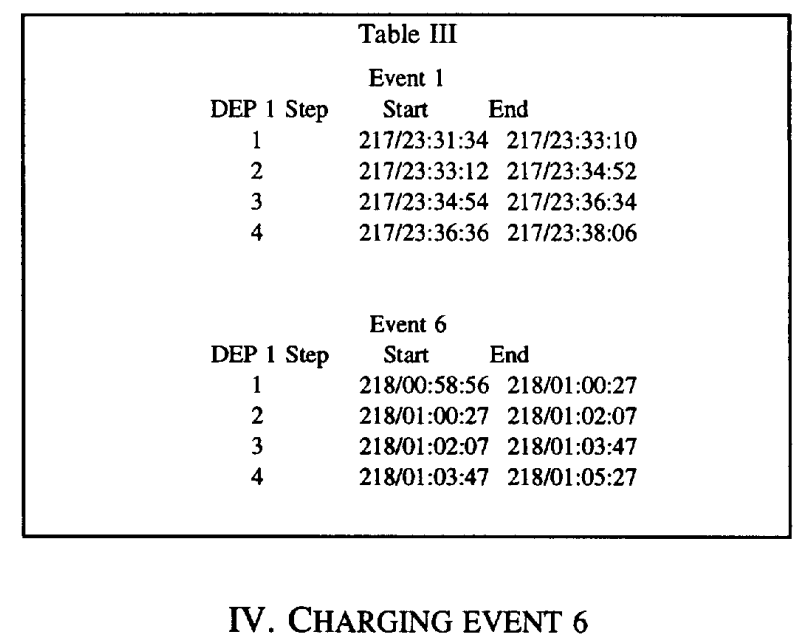

We first consider charging in Event 6 . During this event the tether was deployed to a length of $185.5 \mathrm{~m}$. The Orbiter was in darkness near the geographic equator. The Orbiter's Flash Evaporator System (FES) operated continuously throughout this event, releasing a vapor cloud around the Orbiter. In addition, intermittent Reaction Control Thruster (RCS) firings occurred. Throughout Event 6, the Orbiter was in an attitude with its engine bells in the ram direction, belly to earth, and payload bay to space, essentially flying in a backwards airplane configuration. In this attitude, the SPREE was shadowed from measuring ram ions directly. This wake effect did not preclude the SPREE from measuring electrons. This Orbiter attitude provided the maximum conducting surface exposure to ram allowing maximum current collection from the ambient ionosphere by the Orbiter.

The FPEG emits electrons over an angular cone with an initial half angle of $7.5^{\circ}$. For Event 6 , this emission cone was centered at a pitch angle of approximately $6^{\circ}$. In the SPREE coordinate system, the magnetic field direction was parallel at an azimuthal angle of $202^{\circ}$, in Zone 3 of ESA B. 
During Event 6 there were periods in which the Orbiter was both charged and uncharged. An example of the SPREE data for the period prior to Orbiter charging in Event 6 is shown in Figure 2. In the figure, the differential number flux, as measured by ESA $\mathrm{A}$ in electron Zone 3, is plotted in a grey scale spectrogram for the period from 218/00:59:10 to 218/01:00:40. Energy is plotted on the y axis and time and azimuthal angle are plotted on the $x$ axis. The line plots at the bottom of the figure show the pitch angle and ram angle for the zone as a function of time.

During this period of FPEG operations, SPREE measured intense fluxes of electrons. Typically these fluxes extended up to energies of a couple of hundred $\mathrm{eV}$ although weak fluxes were observed at some angles up to the energy of the FPEG beam. We use the TCVM and DCORE data, from the Event 6 DEP 1 Step 1, in Equation (3) to obtain the result that the Orbiter did not charge to more than a few volts positive during the operation of the FPEG for this case. The Orbiter charged at most to 3 Volts positive, below the SPREE detection range.

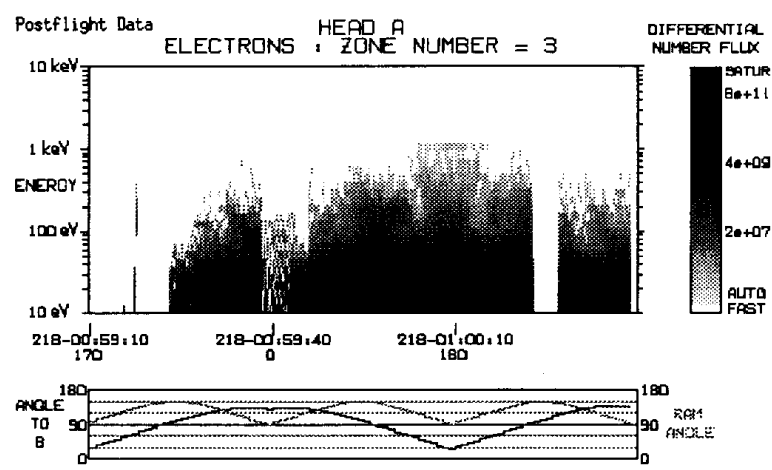

Figure 2 Electron differential number flux spectra measured in ESA A, Zone 3 from 218/00:59:10 to 218/01:00:40.

Gaps in the spectra seen between 218/00:59:39 and 218/00:59:43 are produced by the pulsing of the FPEG at a rate faster than the deflection sweeps of the ESA. The absence of measurable electron fluxes in the SPREE energy range from 218/01:00:22 to 218/01:00:27 results from the termination of the FPEG emission between DEP1 Steps 1 and 2. The FPEG provided a number of short beam emissions starting at 218/00:59:09 and then at 218/00:59:28 began emitting contiguous pulses of varying duty cycles. At 218/00:59:38 the duty cycle was changed to $11 \%$ and held there until 218/00:59:45 after which time the FPEG emitted in a DC mode until it was stopped at 218/01:00:22.

Figure 2 illustrates several points of note. First, although the spectral shape varies with the rotation of the ESAs, intense electron fluxes are seen at all azimuthal angles. Second, the highest electron fluxes are seen at the lowest energies. In general, the electron spectrum is either monotonically decreasing for increasing energy, or shows a peak at an energy of 10 to $20 \mathrm{eV}$ and is monotonically decreasing beyond the peak. Third, the flux shows a consistent pattern in azimuthal angle, i.e. approximately the same spectrum is measured whenever the same azimuthal angle is sampled. This demonstrates that the pattern is relatively time-stable, such that the variations observed with azimuth are variations with respect to pitch angle and angle to the direction of beam propagation.

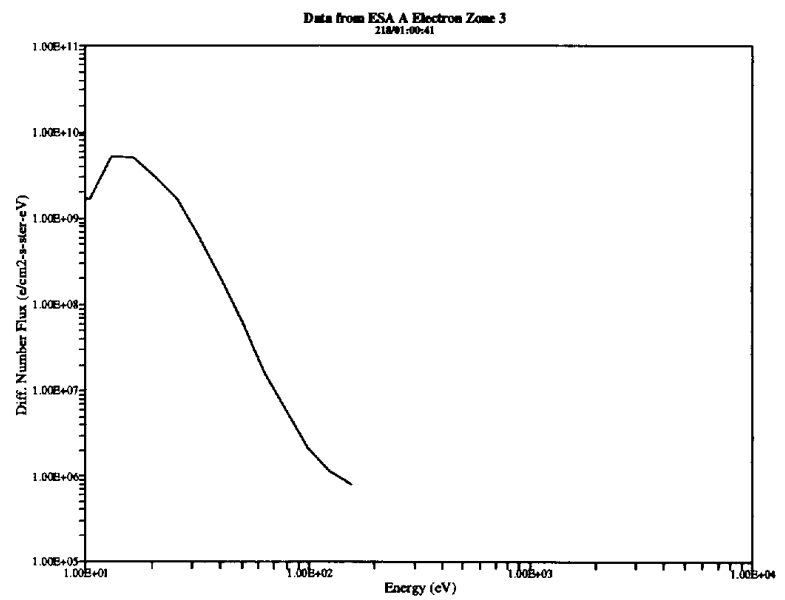

Figure 3 One-second average of the 8 electron spectra measured in ESA A, Zone 3 at 218/01:00:41, plotted as differential number flux versus energy.

A representative spectrum from this DEP 1 Step 1 period is shown in Figure 3. The spectrum is taken from ESA A, Zone 3 at an azimuth angle of $5^{\circ}$ and a pitch angle of $136^{\circ}$ (218/01:00:41). This is a direction well away from the direction of FPEG emission. The spectrum is an average over 8 consecutive spectra. The spectrum is peaked at an energy of approximately $13 \mathrm{eV}$ with a differential number flux at the peak of $5.2 \times 10^{9}$ electrons $/ \mathrm{cm}^{2}-\mathrm{sec}$-ster-eV. The peak in the spectrum is still present if the differential number flux is converted to values of the distribution function indicating a non-thermal distribution. Since we know that the Orbiter is not charged, the peak in the spectrum must be a result of the beam emission process. Integrating over the distribution function for this spectrum and assuming isotropy for the flux gives a number density of approximately $3.8 \times 10^{3}$ electrons $/ \mathrm{cm}^{3}$ and an average energy of $18 \mathrm{eV}$. Such high densities are a significant fraction of the expected night time ionospheric density and indicate a significant heating of a large component of the ambient plasma by the FPEG emissions.

In Figure 4, we have plotted the number flux integrated over the full SPREE energy range for ESA $A$. The integral number flux is plotted as grey scale versus time and the ten angular zones of the detector. The principal point of note is that the integral number flux is reasonably isotropic. Variations across the zones are generally less than a factor of two. The typical levels are $5 \times 10^{10}$ to $2 \times 10^{11}$ electrons $/ \mathrm{cm}^{2}$-ster-sec. 
This plot demonstrates that prior to the positive charging of the Orbiter, the return flux of electrons to the Orbiter was approximately isotropic. Using ESA A's measure of the average total electron number flux in the pre-charging period, and the observation of nearly isotropic return flux, one can calculate the average return current flux to be 0.5 to $1 \mathrm{~mA} / \mathrm{m}^{2}$. If the conductive collecting area of the Orbiter is taken to be on the order of $25 \mathrm{~m}^{2}$, and with a $100 \mathrm{~mA}$ FPEG emission, it appears that the return flux measured by SPREE is not sufficient to compensate for the emitted beam flux. The current balance may well then be achieved mainly through the electrons below $10 \mathrm{eV}$ which are not measured by SPREE or may be done at the FPEG itself.

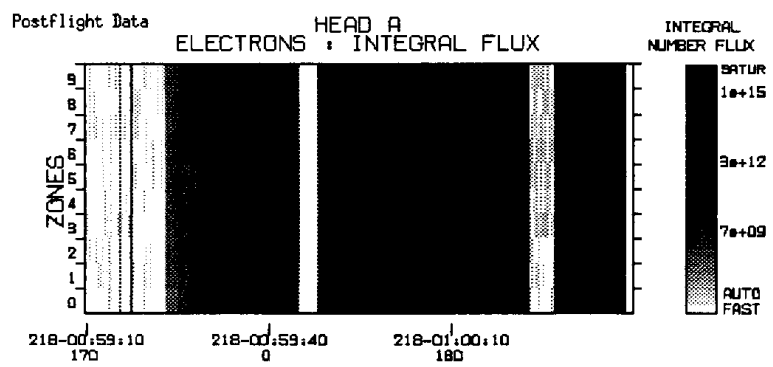

Figure 4 Electron integral number flux from all ten elevation zones from ESA A plotted for the period 218/00:59:10 to 218/01:00:40

For Event 6 the SPREE data show evidence of positive charging of the Orbiter ground from 218/01:01:17 to 218/01:02:04. The onset of the charging coincided with a decrease in the ambient plasma density as measured by RETE.[25] The increase in the level of positive charging is seen to qualitatively follow the decrease in density, i.e., the potential increases as the density decreases. Figure 5 is a grey scale spectrogram of the electron differential number flux from ESA A for Zone 3 for the period from 218/01:00:50 to 218/01:02:20. Note that starting at GMT 218/01:01:00 the electron spectra display a peak at energies above $13 \mathrm{eV}$. The

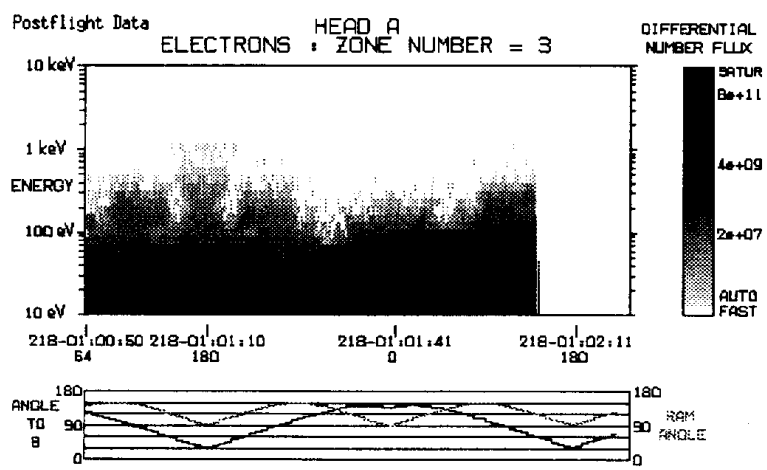

Figure 5 Electron differential number flux spectra in ESA A Zone 3 from 218/01:00:50 to 218/01:02:20 location of the peak moves to higher energies with time reaching values as high as approximately $80 \mathrm{eV}$. This charging period directly corresponds to the shunt $(15 \Omega)$ being inline and FPEG firing. These SPREE peaks are in good agreement with the determined Orbiter potential value, obtained from Equation (5) using TCVM, RETE and SCORE data (see Figure 6).

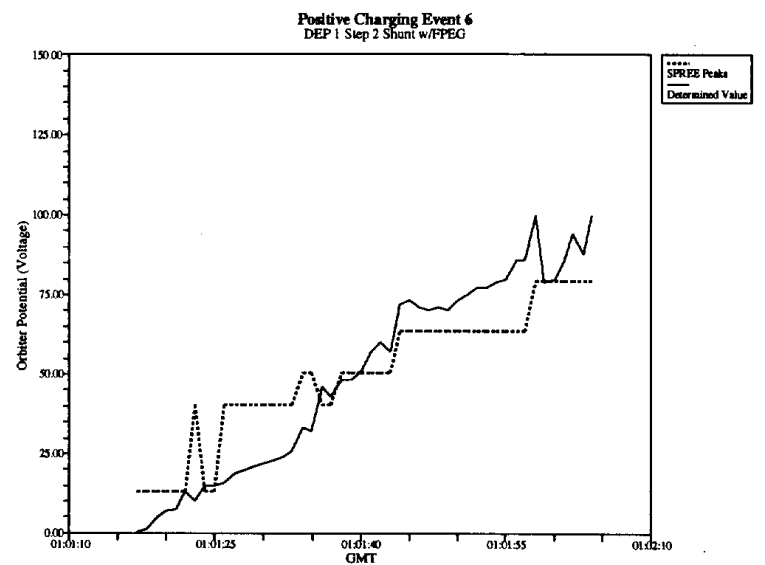

Figure 6 SPREE observed Orbiter potential and determined Orbiter potential for DEP 1 Step 2 of Event 6

That the spectrum is indicative of positive charging of the Orbiter is also demonstrated in Figure 7. In the figure, we have plotted the distribution function of the electrons versus energy on a $\log$ scale for two spectra, one before and one after the start of charging (218/01:00:41 and 218/01:01:41). The two spectra were chosen one minute apart such that they were measured at the same azimuthal and pitch angle. This is necessary since the spectrum is seen to vary significantly in shape and intensity with azimuth. With respect to the spectrum, there are three principal points of note. First, at energies beyond the peak at $50 \mathrm{eV}$ the two spectra have similar shapes simply being displaced by approximately 30 volts. Such a consistent displacement in the spectrum is what would be expected if the electrons fell through a potential drop of 30 volts. This is indicative of positive Orbiter charging for the time 218/01:01:41. Second, below the charging peak there is an additional population of electrons peaked at $15 \mathrm{eV}$ as was present before the charging. In this case the flux at the peak is larger than for the pre-charging spectrum. Assuming isotropy, we integrate over the distribution function for this spectrum, and obtain a number density of $4.6 \times 10^{3} \mathrm{~cm}^{3}$ for this low energy component. Third, the value of the distribution function at the charging peak is $6 \times 10^{-23} \mathrm{sec}^{3} / \mathrm{cm}^{6}$. If the pre-charging spectrum was accelerated by 30 volts then the entire spectrum should be translated by this same amount such that the value at the peak should be $6.5 \times 10^{-22} \mathrm{sec}^{3} / \mathrm{cm}^{6}$. The measured value is lower due to saturation of the detector. The flux at $50 \mathrm{eV}$ predicted from translation of the pre-charging spectrum by $30 \mathrm{eV}$ would require a count rate of greater than a megacycle, beyond the measurement capability of the SPREE. 


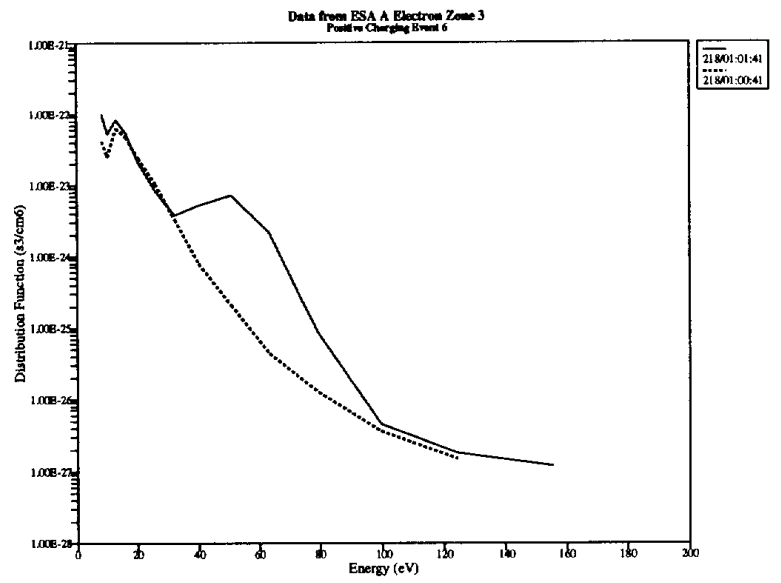

Figure 7 Electron distribution function plotted versus energy, from ESA A, Zone 3 at 218/01:00:41 and at 218/01:01:41.

This saturation effect is also seen in ESA B as shown in Figure 8. In this figure we have only plotted the distribution function for spectra both before and after the onset of positive charging (218/01:00:50 and 218/01:01:50). As for the functions plotted in Figure 7 , for energies above $100 \mathrm{eV}$ the two distribution functions in Figure 8 are simply offset in energy. At this time in the charging, 218/01:01:50, the offset is approximately $80 \mathrm{eV}$. One notes, however, that at $80 \mathrm{eV}$ there is a dip in the value of the distribution function. Again, we attribute this dip to saturation of the detector. The effect of this saturation is that it is very difficult to determine the true thermal and charging peaks in the spectra. For the TSS reflight, the ESAs' geometric factors will be adjusted to prevent this saturation effect from hindering our measurements during both FPEG and EGA emissions.

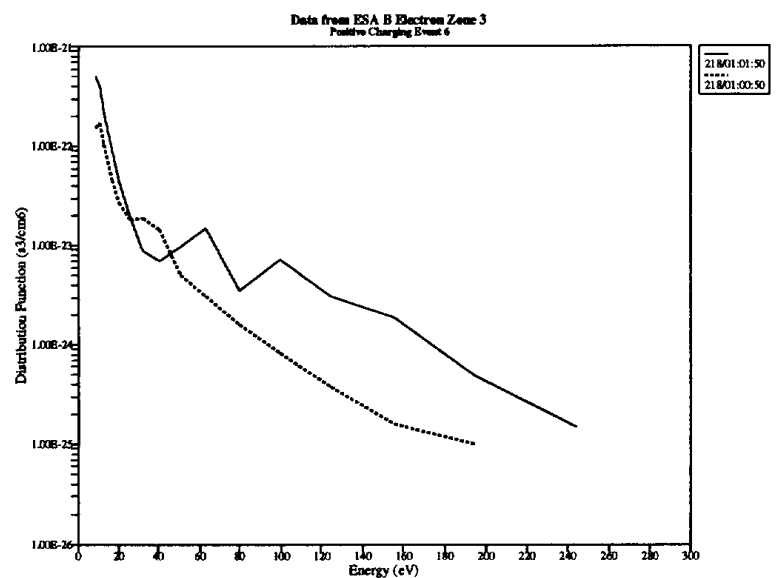

Figure 8 Electron distribution function plotted versus energy, from ESA B, Zone 3, at 218/01:00:50 and at 218/01:01:50

\section{Charging EVENT 1}

For the Charging Event 1, the Orbiter was again in darkness with the tether being deployed during the event from a distance of 105.3 to 148.9 meters. During this period the Orbiter was oriented with the engine bells into the ram and the payload bay to space. The Orbiter was pitched up with respect to the velocity vector by $40^{\circ}$ degrees such that SPREE was deep in the wake region. In Event 1, the FPEG does not escape the Orbiter when fired as it did in Event 6 . It hits the starboard payload bay door to the rear of the Orbiter. This event differs from the Event 6 in several ways. First, the Orbiter is charged positively throughout the event both during DC and pulsed operation of the FPEG. For this reason, we cannot compare charging and non-charging spectra. Second, the level of charging appears to be independent of whether FPEG is pulsing or DC. Third, while intense electron fluxes are observed for Event 1 they are not as large as those seen in the Event 6 and there is no indication of saturation of either ESA A or B. Lastly, the intensity of the electron flux at energies below the charging peak varies by an order of magnitude or more during the event.

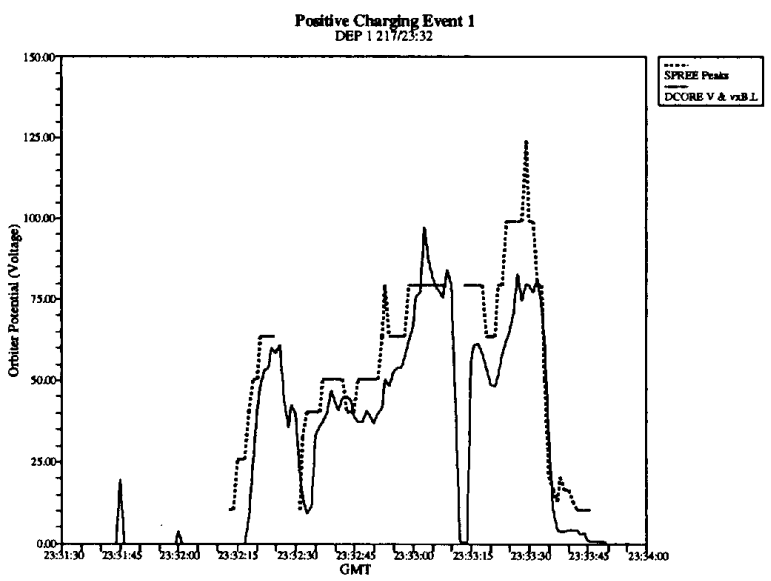

Figure 9 SPREE observed Orbiter potential and determined Orbiter potential for DEP 1 Steps 1 and 2 of Event 1

That the Orbiter was charged is shown in Figures 9 and 10. In Figure 9 we have plotted the SPREE spectral peaks with the Orbiter potential as determined from Equation (3) using DCORE measured tether voltage and calculated $v x B \cdot L$ voltage. Gaps in the SPREE spectral peaks plot are periods of time when the Orbiter potential was below the SPREE detection range or was zero. Figure 10 is a grey scale spectrogram of the electron data from Event 1 for the period from 217:23:32:00 to 217:23:33:30 from ESA A electron zone 6. As for Event 6, the electron spectra are peaked at energies between 10 and $100 \mathrm{eV}$.

Two typical differential number flux spectra are shown in Figure 11 (217/23:32:18 and 217/23:33:02). As before, these 


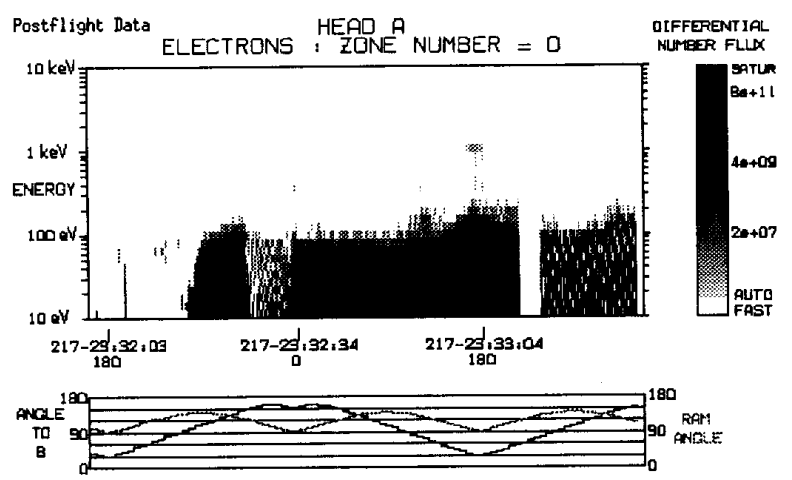

Figure 10 Electron differential number flux spectra measured in ESA A, Zone 0 from 217/23:32:00 to 217/23:33:30

are averages over 8 consecutive fast sweep spectra. The two spectra are for cases where the charging peak is at approximately $40 \mathrm{eV}$ and $100 \mathrm{eV}$, respectively. For both spectra the flux fall off rapidly at energies above the peak indicating a relatively cold parent population. This parent population is much colder than that seen in Event 6, where measurable fluxes were seen in more energy channels above the charging peak energy channel. At lower energies, the $\mathbf{4 0}$ Volt charging spectrum shows a peak at approximately $10 \mathrm{eV}$. The 100 Volt charging spectrum shows a much more intense low energy component with no peak within the energy range of the SPREE. This part of the spectrum can be fit to a power law of the form $\mathrm{j}(\mathrm{E})=\mathrm{aE}^{\mathrm{b}}$ where in this case $\mathrm{b}=-3.39$ and $\mathrm{a}$ $=2.807 \times 10^{13}$, where $\mathrm{E}$ is in $\mathrm{eV}$ and differential number flux is in electrons $/ \mathrm{cm}^{2}$-ster-sec-eV.

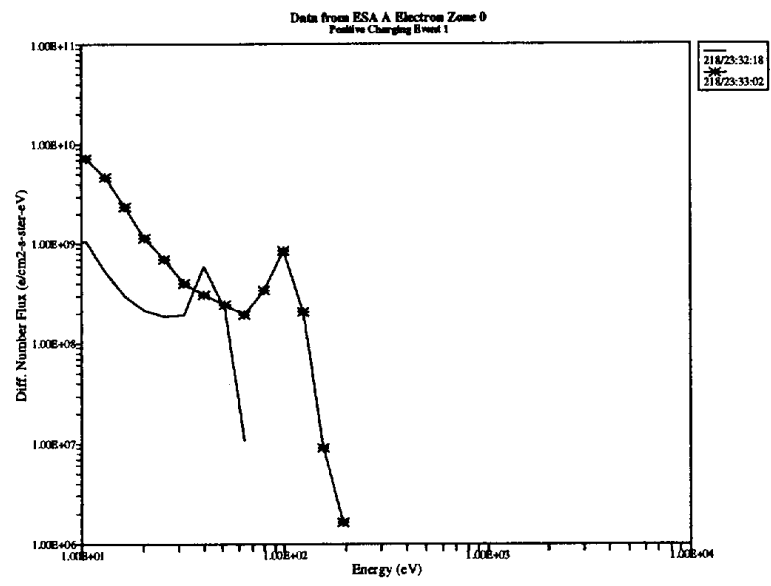

Figure 11 One-second average of the 8 electron spectra measured ESA A, Zone 0, at 217/23:32:18 and at 217/23:33:02, plotted as differential number flux versus energy

Since the measurements in this case appear to be unsaturated we can use them to investigate the directionality of the flux at the peak of the charging spectrum. An example of this is shown in Figure 12. In the figure the variation in the differential number flux across the 10 zones of the detection fan is plotted for the $40.4 \mathrm{eV}$ channel at 217/23:32:34. At this time, the charging peak in the spectrum was at this energy as can be seen in Figure 10. The ESA A detection fan was at an azimuth of $8^{\circ}$ looking out over the Orbiter's port wing. The values are an average over 8 consecutive ESA sweeps. The intensity is seen to vary by more than a factor of 5 across the zones with the lowest flux in Zone 1 and the highest flux in Zone 8. By contrast, the flux is found to be close to isotropic across the zones for the energy channels below the peak. In general, the flux at the charging peak is not isotropic with the direction of maximum flux varying with azimuth and with the level of the charging. Further analysis is being performed to determine whether the anisotropy is produced by the charging or if it arises from secondary emission of electrons from the Orbiter's surfaces bombarded by the non-escaping FPEG. The fact that the flux is isotropic across the zones for energies below the charging peak demonstrates that the effect is not produced by shadowing of the particles by the Orbiter's physical structure.

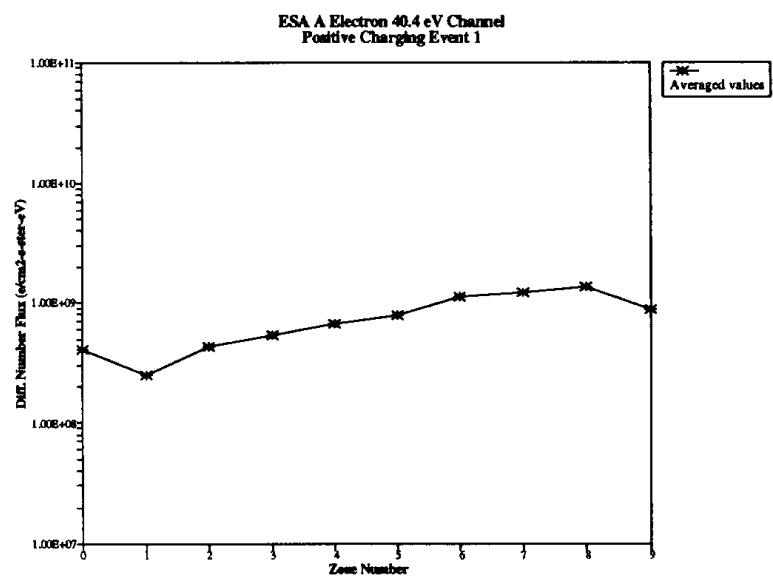

Figure 12 Differential number flux in the $40.4 \mathrm{eV}$ channel plotted as a function of the ESA elevation zones, at 217/23:32:34

As a last point, we note that in this charging event a period of significant modulation of the electrons was observed in the LF Autocorrelator data. The modulations were observed between 217/23:32:33 and 217/23:32:35 when the Orbiter was charged to approximately 40 volts. The LF Autocorrelator data are shown in Figures 13a and 13b. At this time the LF Autocorrelator was operating to detect modulations in the 0 to $1.25 \mathrm{KHz}$ range. In the figures we have plotted two line graphs. The plot in Figure 13a shows the ACF as determined by the LF Autocorrelator and the plot in Figure 13b is the Fast Fourier Transform of the ACF. These are data for electrons measured in ESA A electron Zone 8 and at the 40 volt charging peak. In the ACF, one notes a series of distinct periodic peaks. In the FFT, these are seen to produce a principal peak in frequency at $312 \mathrm{~Hz}$. Further studies are underway to determine the nature of this wave-particle interaction in the charging observed. 


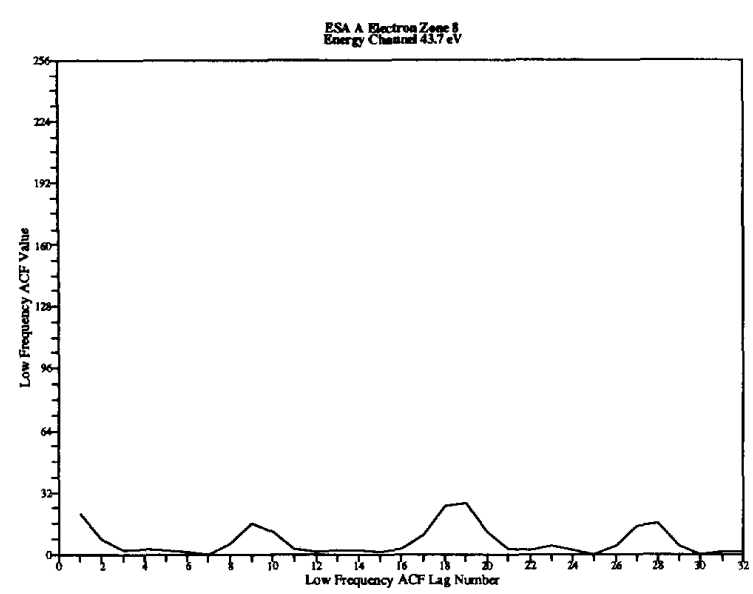

Figure 13a LF ACF values versus Lag

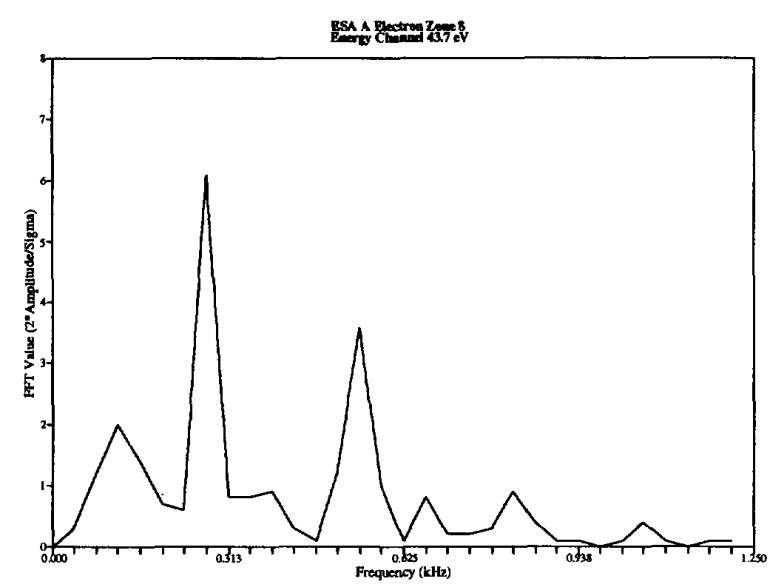

Figure 13b Fast Fourier Transform of LF ACF data versus frequency

\section{SUMMARY}

In this paper, we have reported on observations of positive charging of the Orbiter during the deployed phase of the TSS-1. The charging is observed to occur when the Orbiter was in darkness, during periods of low ionospheric density and the SETS FPEG was emitting a $1 \mathrm{keV}, 100 \mathrm{~mA}$ electron beam. The charging occurred when the ambient plasma density was too low to provide a current to match the FPEG emission. For the cases where the ambient plasma temperature was approximately $0.1 \mathrm{eV}$, and assuming a conducting area of the Orbiter of approximately $25 \mathrm{~m}^{2}$, the positive charging occurred for densities below $6 \times 10^{5}$ electrons $/ \mathrm{cm}^{3}$.

The positive charging of the Orbiter is seen in the SPREE data as a peak in the electron spectrum produced by the acceleration of the pre-existing electrons by the electric field created by the charging. Prior to the charging, the electron population in the energy range above $10 \mathrm{eV}$ had already been greatly enhanced by the operation of the FPEG. The charging is seen as an acceleration of this already enhanced and relatively hot electron population. Intense fluxes of electrons were observed at low energy for both the charged and uncharged Orbiter. This low energy component tended to be isotropic with densities as high as $4.6 \times 10^{3}$ electrons $/ \mathrm{cm}^{3}$. The return flux at the charging peak was anisotropic, with the anisotropy varying with the level of charging and the pitch angle.

\section{ACKNOWLEDGEMENTS}

The authors wish to acknowledge the work of N. Bonito, C. Roth, K. Bounar, and W. McNeil in developing the graphics software for the display and analysis of SPREE data. We also wish to thank J.O. McGarity, D.J. Sperry, A.W. Everest, A.C. Huber, E. Holeman and J.A. Pantazis for all of their efforts in the flight of SPREE. We also thank S. Williams for his $v x B \cdot L$ voltage calculations.

\section{REFERENCES}

[1] P.M. Banks, P.R. Williamson, and K.-I. Oyama, "Electrical Behavior of a Shuttle Electrodynamic Tether System (SETS)," Planet. Space Sci., Vol. 29, p. 139-147, 1981

[2] M. Dobrowolny, "The TSS Project: electrodynamics of long metallic tethers in the ionosphere," Il Nuovo Cimento, Vol. 10, Number 3, 1987

[3] M. Dobrowolny, and N.H. Stone, "A Technical Overview of TSS1: The First Tethered Satellite System Mission," accepted for publication in Il Nuovo Cimento, April 1993

[4] P.M. Banks, et. al., "The Shuttle Electrodynamic Tether System (SETS)," accepted for publication in Il Nuovo Cimento, April 1993

[5] N. Singh, K.H. Wright, Jr., and N.H. Stone, eds., Current Collection from Space Plasmas, NASA-CP-3089, 1990

[6] W.J. Raitt,, et. al., "Charge-2B: A Sounding Rocket Experiment to Study the Generation and Propagation of VLF Waves by a Modulated Electron Beam," submitted to J. Spacecr. Rockets, 1993

[7] B. Maehlum, et. al., "Studies of electrical charging of the tethered electron accelerator mother-daughter rocket Maimik," Geophys. Res. Lett., Vol. 15, p. 725, 1988

[8] N.B. Myers, et. al., "Vehicle Charging Effects During Electron Beam Emission from Charge-2 Experiment," J. Spacecr. Rockets, Vol. 27, p. $25-37,1990$

[9] T. Neubert, et. al., "The sheath structure around a negatively charged rocket payload," J. Geophys. Res., Vol. 95, p. 6155-6165, 1990

[10] J.R. Winckler, et. al., "ECHO-7: An electron beam experiment in the magnetosphere," EOS Trans. of the AGU, Vol. 70, p.657-668, 1989 
[11] S. Sasaki, et. al., "Results from a series of tethered rocket experiments," J. Spacecr. Rockets, Vol 24, p. 444-453, 1987

[12] H.G. James and B.A. Whalen, "Oedipus-A: space research with a new tether," EOS Trans. of the AGU, Vol. 72, p. 137, 1991

[13] P.M. Banks, P.M., et. al., "Results from the Vehicle Charging and Potential Experiments on STS-3," J. Spacecr. Rockets, Vol. 24, p. $138-149,1987$

[14] P.R. Williamson, P.M. Banks, and W.J. Raitt, "Shuttle Electrodynamic Tether System," Space Tethers for Science in the Space Station Era, Società Italiana di Fisica, Conference Proceedings No. 14,1988

[15] D.C. Thompson, et. al., "TSS-1 Orbiter Current and Voltage Experiments," AIAA Paper 93-0702, 1993

[16] C. Bonifazi, et. al., "TSS Core Equipment: A High Perveance Electron Generator for the Electrodynamic Mission," Space Tethers for Science in the Space Station Era, Società Italiana di Fisica, Conference Proceedings No. 14, 1988

[17] C. Bonifazi, et. al., "TSS Core Equipment," accepted for publication to Il Nuovo Cimento, April 1993

[18] J.G. Laframboise and L.J. Sonmor, "Current Collection by Space Probes in the Magnetosphere: a Review," J. Geophys. Res., Vol. 98, No. A1, p. 337-357, 1 Jan 93

[19] I. Langmuir, and K.B. Blodgett, "Currents Limited by Space Charge between Concentric Spheres," Phys. Rev., Vol. 24, p. 49, 1924

[20] L.W. Parker and B.L. Murphy, "Potential Buildup on an Electron-Emitting Ionospheric Satellite," J. Geophys. Res., Vol. 72, No. 5, March 1, 1967

[21] J.O. McGarity, et. al., "Compact Ion/electron analyzer for spaceflight or laboratory use," Rev. Sci. Instrum., Vol. 63, March 1992, p. 1973

[22] M.R. Oberhardt, et. al., "The Shuttle Potential and Return Electron Experiment," accepted for publication in Il Nuovo Cimento, April 1993

[23] V.A. Davis, I. Katz, T.T. Luu, and M.R. Oberhardt, "Predicted Potentials and Currents for TSS-1," AIAA Paper No. 92-0574

[24] M. Dobrowolny, et. al., "The Research on Electrodynamic Tether Effects (RETE) Experiment," accepted for publication in Il Nuovo Cimento, April 1993

[25] B.E. Gilchrist, C. Bonifazi, M. Dobrowolny, private communication, Dec 92 